\title{
Siderophore Production by Rhizosphere Biological Control Bacteria Brevibacillus brevis GZDF3 of Pinellia ternata and Its Antifungal Effects on Candida albicans
}

\author{
Miaomiao Sheng ${ }^{1 \dagger}$, Huake Jia ${ }^{1 \dagger}$, Gongyou Zhang ${ }^{1}$, Lina Zeng ${ }^{1,2}$, Tingting Zhang ${ }^{1,2}$, Yaohang Long ${ }^{1,2}$ \\ Jing Lan ${ }^{1,2}$, Zuquan $\mathrm{Hu}^{1,2}$, Zhu Zeng ${ }^{1,2,3}$, Bing Wang ${ }^{1,2 *}$, and Hongmei Liu ${ }^{1,2 *}$ \\ ${ }^{1}$ Engineering Research Center of Medical Biotechnology, Guizhou Medical University, Guiyang 550025, Guizhou, \\ P.R. China \\ ${ }^{2}$ Immune Cells and Antibody Engineering Research Center of Guizhou Province, Key Laboratory of Biology and \\ Medical Engineering, School of Biology and Engineering, Guizhou Medical University, Guiyang 550025, Guizhou, \\ P.R. China \\ ${ }^{3}$ School of Basic Medical Sciences, Guizhou Medical University, Guiyang 550025, Guizhou, P.R. China
}

Brevibacillus brevis GZDF3 is a gram-positive, plant growth-promoting rhizosphere bacterium (PGPR) isolated from the rhizosphere soil of Pinellia ternata (an important herb in traditional Chinese medicine). The GZDF3 strain produces certain active compounds, such as siderophores, which are the final metabolite products of non-ribosomal peptide synthetase (NRPS) and independent non-ribosomal peptide synthetase (NIS) activity. With the present study, we attempted to investigate the siderophore production characteristics and conditions of Bacillus sp. GZDF3. The antibacterial activity of the siderophores on pathogenic fungi was also investigated. Optimal conditions for the synthesis of siderophores were determined by single factor method, using sucrose $15 \mathrm{~g} / \mathrm{l}$, asparagine $2 \mathrm{~g} / \mathrm{l}, 32^{\circ} \mathrm{C}$, and $48 \mathrm{~h}$. The optimized sucrose asparagine medium significantly increased the production of siderophores, from $27.09 \%$ to $54.99 \%$. Moreover, the effects of different kinds of metal ions on siderophore production were explored here. We found that $\mathrm{Fe}^{3+}$ and $\mathrm{Cu}^{2+}$ significantly inhibited the synthesis of siderophores. The preliminary separation and purification of siderophores by immobilized-metal affinity chromatography (IMAC) provides strong antibacterial activity against Candida albicans. The synergistic effect of siderophores and amphotericin B was also demonstrated. Our results have shown that the GZDF3 strain could produce a large amount of siderophores with strong antagonistic activity, which is helpful in the development of new biological control agents.

Received: October 29, 2019 Accepted: February 18, 2020

First published online: February 21, 2020

${ }^{*}$ Corresponding authors B.W.

Phone: +86-851- 88174039 Fax: $+86-851-88174039$ E-mail:WangBing_gmu_edu@ 163.com

H.L.

E-mail: hmliu@gmc.edu.cn

${ }^{\dagger}$ These authors contributed equally to this work.

pISSN 1017-7825 elSSN 1738-8872

Copyright(C) 2020 by The Korean Society for Microbiology and Biotechnology
Keywords: Brevibacillus brevis GZDF3, siderophore, Candida albicans, Pinellia ternata

\section{Introduction}

Iron is essential for the growth and survival of most microorganisms. However, in the natural environment, iron is mainly present in the form of insoluble $\mathrm{Fe}^{3+}\left(10^{-18} \mathrm{M}\right.$ at $\mathrm{pH}$ 7.0). Therefore, it is difficult to obtain available iron from the surroundings for microorganisms $[1,2]$. One of the most common strategies developed by bacteria, fungi and plants is the production of siderophores (from Greek, meaning "iron carriers"). Siderophores are natural iron chelators, with low molecular weight secondary metabolites $(400-2,000 \mathrm{Da})$, and high affinity for $\mathrm{Fe}^{3+}$. They are secreted to acquire iron as needed by microorganisms [3-5]. So far, more than 500 kinds of siderophores have been reported, and these can be divided into hydroxamaces, catecholates, carboxylates and various other types according to the characteristics of their coordination structure [6]. In iron deficient environments, microorganisms secrete synthetic siderophores to obtain iron and maintain their growth. Some plant growth-promoting rhizobacteria (PGPR) can synthesize siderophores, compete with pathogens to obtain iron, and inhibit the growth of pathogenic microbes [7, 8].

We previously reported that Brevibacillus brevis GZDF3 (CGMCC No. 10121) was isolated from the rhizosphere soil of Pinellia ternata. It was a new biocontrol bacterium found via hot screen method isolated from the rhizosphere soil of $P$. ternata in Guizhou Province, China. B. brevis GZDF3 is used not only for control of pathogenic organisms such as Pectobacterium carotovorum subsp. carotovorum, but also the pathogenic fungi Fusarium solani and Fusarium oxysporum. The GZDF3 strain has strong antagonistic activity against other 
pathogenic microbes as well. Therefore, it has great potential for biocontrol [9]. In recent years, siderophores have drawn much attention due to their potent roles in the medical industry, agriculture, and environmental science [10]. For instance, siderophore enterobactin of Escherichia coli promotes mitochondrial iron uptake and the development of Caenorhabditis elegans via interaction with its ATP synthase [11]. Our previous research reported that B. brevis GZDF3 can produce catechol-type siderophores and has a strong antagonistic effect on $F$. oxysporum. However, factors that affect the production of siderophores and the antifungal effect against Candida albicans of B. brevis GZDF3 have not yet been reported [12].

Diverse factors, such as carbon source, nitrogen source, $\mathrm{pH}$, temperature and metal ions have been reported to affect the synthesis of siderophores [13]. Moreover, maximum siderophore production conditions vary according to strain type $[13,14]$. Bendale reported that maximum siderophore production could be achieved when sucrose was used as a carbon source by Streptomyces fulvissimus. Whereas in Santos's study, glycerol was proven to be the favorite carbon source for the growth of Bacillus megatherium [13, 14]. Maximum siderophore production of Bacillus sp. PZ-1 was observed in the glucose concentration of $21.84 \mathrm{~g} / 1$ [15]. The $\mathrm{pH}$ is also a crucial factor in siderophore production. Generally, a neutral $\mathrm{pH}$ is favorable for microorganisms to produce the maximum amount of siderophores in fluorescent pseudomonads [16]. In Pseudomonas syringae strain BAF.1, maximum siderophore production was achieved with glucose as a carbon source and asparagine as a nitrogen source, at $30^{\circ} \mathrm{C}$ and $\mathrm{pH} 7.0[17]$.

It is important to note that many metal ions have shown the capacity to affect siderophore production [18, 19]. It was reported that $\mathrm{Zn}$ ion increased the siderophore production whereas $\mathrm{Cu}$ ion decreased it [20]. Addition of $\mathrm{Cd}$ also enhanced the production of siderophore in iron-limited cultures [19]. In fungal strains, the maximum siderophore production was detected at $\mathrm{Zn}$ ion concentration of $150 \mu \mathrm{g} / \mathrm{ml}[20,21]$. Moreover, the Pb ion could also stimulate PZ-1 strain siderophore production [15, 22].

C. albicans is an important opportunistic pathogen that can cause a variety of human infections, and the current treatment involves the use of antifungal agents, such as polyenes and azoles [23]. Ergosterol is a neutral lipid in fungal membranes, and being critical for many cell processes [24], the destruction of its synthesis has become the focus of antifungal therapy. Both types of antifungal agents cause the disorder of plasma membrane structure and function by combining ergosterol (polyenes, e.g., amphotericin B) or inhibiting the enzyme lanosterol 14ademethylase, which is involved in ergosterol biosynthesis (azoles, e.g., fluconazole) [25]. Iron is an essential nutrient for all living cells. There is increasing evidence that interfering with the iron homeostasis of C. albicans can improve its antifungal response. It has been shown that iron-chelating agents can improve the activity of azole. The iron carrier, as an iron-chelating agent, is a potential new antibiotic and bacteriostatic agent [26]. Kimley has reported, that the new iron-chelating agent (DIBI) has stronger growth inhibition effect with various azole combinations than that of albicides alone. It greatly prolongs the inhibition of C. albicans cell proliferation [27]. In this study, we optimized the composition of B.brevis GZDF3 medium and related culture conditions and investigated the effect of metal ions on the synthesis of iron carrier. The antibacterial activity of iron carrier against C. albicans was preliminarily verified, and the synergistic effect of siderophores with amphotericin B was demonstrated. Herein, these results provide new possibility for further understanding the mechanism of action of GZDF3 on C. albicans and its application in the cultivation of Pinellia ternata, while also serving as a useful reference for fighting fungal infection.

\section{Materials and Methods \\ Microorganism Strains and Medium}

B. brevis GZDF3 and C. albicans are preserved in the Department of Biotechnology, College of Biology and Engineering, Guizhou Medical University. The strain was subsequently grown on Luria-Bertani (LB) medium (10 g/l tryptone, $5 \mathrm{~g} / \mathrm{l}$ yeast extract, $10 \mathrm{~g} / \mathrm{l} \mathrm{NaCl})$ or Potato Dextrose Agar (PDA) medium (200 g/l potato, $20 \mathrm{~g} / \mathrm{l}$ glucose). The bacterium was maintained in glycerol and kept at $-80^{\circ} \mathrm{C}$. To screen for siderophore production, we chose the optimized fermentation sucrose-asparagine (SA) medium ( $20 \mathrm{~g} / \mathrm{l}$ sucrose, $2 \mathrm{~g} / \mathrm{l} \mathrm{L}$-asparagine anhydrous, $1 \mathrm{~g} / \mathrm{l} \mathrm{KH}_{2} \mathrm{PO}_{4}$, and $1 \mathrm{~g} / 1 \mathrm{MnSO}_{4} \cdot 7 \mathrm{H}_{2} \mathrm{O}$ ).

\section{Chrome Azurol S (CAS) Liquid Detection}

The strain GZDF3 was activated and the bacterial culture solution was prepared. The bacterial culture solution was added into $150 \mathrm{ml}$ of SA medium at a dose of $5 \%$, and cultured at $28^{\circ} \mathrm{C}$ and $200 \mathrm{rpm}$ for $36 \mathrm{~h}$, followed by centrifugation at $12,000 \times g$ for $10 \mathrm{~min}$. The supernatant was removed by filtration using a $0.22 \mu \mathrm{m}$ microporous membrane. Next, we mixed $1 \mathrm{ml}$ of fermentation broth with an equal volume of CAS blue detection solution. The siderophore production was determined by CAS liquid test. The SU was calculated by the following formula: $\mathrm{SU}=$ $[(\mathrm{Ar}-\mathrm{As}) / \mathrm{Ar}] \times 100 \%$. Where Ar represents the absorbance of reference (uninoculated broth), and As is the absorbance of the sample (culture supernatant) at $630 \mathrm{~nm}$ [28].

\section{SA-CAS Plate Assay}

The universal CAS assay (Schwyn and Neilands 1987) was modified to test the production ability of microorganisms with siderophore-type, iron-binding compounds in solid medium avoiding growth inhibition caused by the toxicity of CAS-blue agar medium. CAS-blue agar $(100 \mathrm{ml})$ was prepared according to Schwyn and Neilands (1987). Petri dishes (10 cm in diameter) were prepared with $30 \mathrm{ml}$ of appropriate medium for culturing each strain. After solidifying, the medium was cut into halves, one of which was replaced by CAS-blue agar $(15 \mathrm{ml})$. The halves containing culture medium (SA) were inoculated with strains taken from stock cultures. The inoculum was placed as far as possible from the borderline between the two media. Then, the plate was incubated 
for $48 \mathrm{~h}$ at $28^{\circ} \mathrm{C}$. The CAS reaction was determined by measuring the position or distance of the advancing colorchange front in the CAS-blue agar, starting from the borderline between the two halves during all incubation times [28].

\section{Optimization of the Cultural Conditions for Siderophore Production}

A single-factor experiment was adopted to evaluate the effect of physicochemical parameters (fermentation time, culture $\mathrm{pH}$, fermentation temperature, carbon source, nitrogen source and metal ion) on cell growth and siderophore production of GZDF3 strain. Cell growth was measured at $600 \mathrm{~nm}$ using a spectrophotometer [28]. The siderophore production was determined by CAS liquid test. The cell growth and siderophore production were measured as a function of fermentation time at $0,6,12,18,24,30,36,42,48,54$, and $60 \mathrm{~h}$. The $\mathrm{pH}$ was adjusted to 4.0, 5.0, 6.0, 7.0, 8.0, 9.0, and 10.0, respectively. Fermentation temperature was set from $23^{\circ} \mathrm{C}$ to $37^{\circ} \mathrm{C}$. Fructose, maltose, glucose, mannose, glycerol and xylose were used at a concentration of $20.0 \mathrm{~g} / \mathrm{l}$, replacing sucrose as a carbon source in SA medium. In addition, glutamic acid, lysine, tyrosine, tryptophan, and proline were used at a concentration of $2.0 \mathrm{~g} / \mathrm{l}$, substituting for asparagine as a nitrogen source in SA medium. The effect of metal ion was tested by supplementing SA medium with $\mathrm{FeCl}_{3} \bullet 6 \mathrm{H}_{2} \mathrm{O}, \mathrm{CuSO}_{4}, \mathrm{MnSO}_{4}$, and $\mathrm{ZnSO}_{4} \cdot 7 \mathrm{H}_{2} \mathrm{O}$ at a concentration of $0,5,10,20,50,100,200,400$, and $800 \mu \mathrm{mol} / \mathrm{l}$, respectively. All culture flasks were incubated in a shaker at $200 \mathrm{rpm}$ and $28^{\circ} \mathrm{C}$ for $48 \mathrm{~h}$, except for temperature experiments. The siderophore production was then quantified according to the above method.

\section{Preliminary Purification of Siderophore}

After culturing for $48 \mathrm{~h}$ at $30^{\circ} \mathrm{C}$ and $200 \mathrm{rpm}$, the liquid culture was centrifuged at $1,000 \times g$ for $10 \mathrm{~min}$ and the culture filtrate was harvested. Then, the culture filtrate was mixed with 4 volumes of ethanol, precipitated overnight, concentrated by a rotary vacuum evaporator, resuspended in deionized water, and subjected to further study. The filtrate was used as the crude fermentation siderophore solution. The crude solution was then purified by $\mathrm{Fe}(\mathrm{III})$-IMAC column (HiTrap Chelating HP/1 ml). The purified siderophore solutions were used in the antifungal experiments [29].

\section{Antagonistic Activity Analysis of the Siderophore Solution}

In this study, the punch method (agar cup method) was used to investigate the antifungal activity of the siderophore solution [30]. SA medium was inoculated with GZDF3, followed by incubation at $32^{\circ} \mathrm{C}$ for $48 \mathrm{~h}$. Next, crude and purified siderophore solutions were obtained as described above.

PDA plates were prepared and used for antimicrobial screening. Overnight broth cultures of the test organisms were seeded on plates. After that, wells approximately $8 \mathrm{~mm}$ in diameter and $2 \mathrm{~mm}$ deep were made on the surface of the solid medium using a sterile borer. Each well was subsequently filled with $100 \mu \mathrm{l}$ of test sample and labeled with a marker. Sterile SA medium was used as negative control, while ketoconazole was used as positive control. The test samples were $1 \mathrm{mg} / \mathrm{ml}, 5 \mathrm{mg} / \mathrm{ml}, 10 \mathrm{mg} / \mathrm{ml}$ of ketoconazole, purified siderophore, $\mathrm{Fe}^{3+}$-treated crude fermentation medium, GZDF3 solution fermented in SA medium for $36 \mathrm{~h}$ and GZDF3 solution fermented in the optimized SA medium for $48 \mathrm{~h}$. All plates were incubated at $28^{\circ} \mathrm{C}$ for $36 \mathrm{~h}$. Then, a photo was taken and analyzed. The zones of inhibition on the plate were measured $(\mathrm{mm})$ by cross measurement method to evaluate the antifungal activity.

The inhibitory effects of different concentrations of siderophore on C. albicans at different times were measured by microplate reader, and time-kill curves were drawn out. C. albicans was cultured in the potato dextrose (PDB) liquid medium. Different concentrations of siderophore $(2,500,1,250,625,312.5,156.3,78.1,39.1,19.5 \mu \mathrm{g} / \mathrm{ml})$ were purified by Fe (III)-IMAC column and added to the medium. The PDB medium-only group (Medium) was used as a negative control. C. albicans with PDB medium (Bacteria Solution) was used as a control group. Amphotericin B $(19.5 \mu \mathrm{g} / \mathrm{ml})$ (AMP) was used as a positive control. Then, samples were detected by microplate reader at different time points $(0,2,4,6,8,10,12,14,16,18,20,22,24,26 \mathrm{~h})$. The $\mathrm{OD}_{600}$ value represents the bacteria concentration of C. albicans. Data are representative of three independent experiments.

\section{Checkerboard Assay}

The checkerboard assay for siderophores and amphotericin B was performed on the 96-well microtiter plate with the concentration of siderophore ranging from $2,040 \mu \mathrm{g} / \mathrm{ml}$ to $0 \mu \mathrm{g} / \mathrm{ml}$, and the concentration of amphotericin B ranging from $200 \mu \mathrm{g} / \mathrm{ml}$ to $0 \mu \mathrm{g} / \mathrm{ml}$, multiple proportion dilution. Each well contained the starting inoculum of approximately $1.5 \times 10^{8} \mathrm{CFU} / \mathrm{ml}$, and the predefined concentration of each drug in the total volume of $50 \mu \mathrm{l}$. The 96 -well plate was oscillated at $28^{\circ} \mathrm{C}$ for $150 \mathrm{rpm} / \mathrm{min}$ for $24 \mathrm{~h}$, the turbidity of the medium was observed, and the absorbance of $\mathrm{OD}_{492} \mathrm{~nm}$ was measured. Each combination was performed in triplicate. The fractional inhibitory concentration index (FICI) for the two drugs was calculated as follows:

$$
\text { FICI }=\frac{\text { MIC (siderophore) }}{\text { MIC (siderophore) com }}+\frac{\text { MIC (amphotericin A }) \text { com }}{\text { MIC (amphotericin A) com }}
$$

The interaction between the two drugs was interpreted as synergistic if the FICI was $\leq 0.5$, indifferent if it was $>$ 0.5 and $\leq 4$, and antagonistic if it was $>4[31]$. 
A

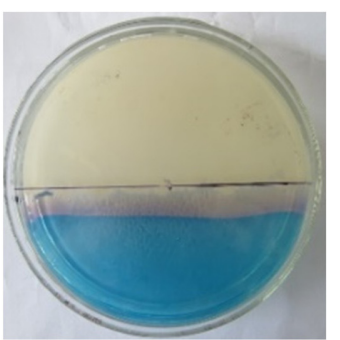

SA-CAS agar plate
B

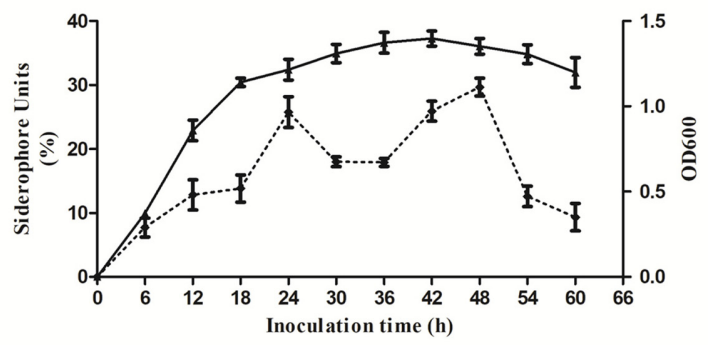

C

-๑. SU $(\%)$

$\rightarrow$ Growth
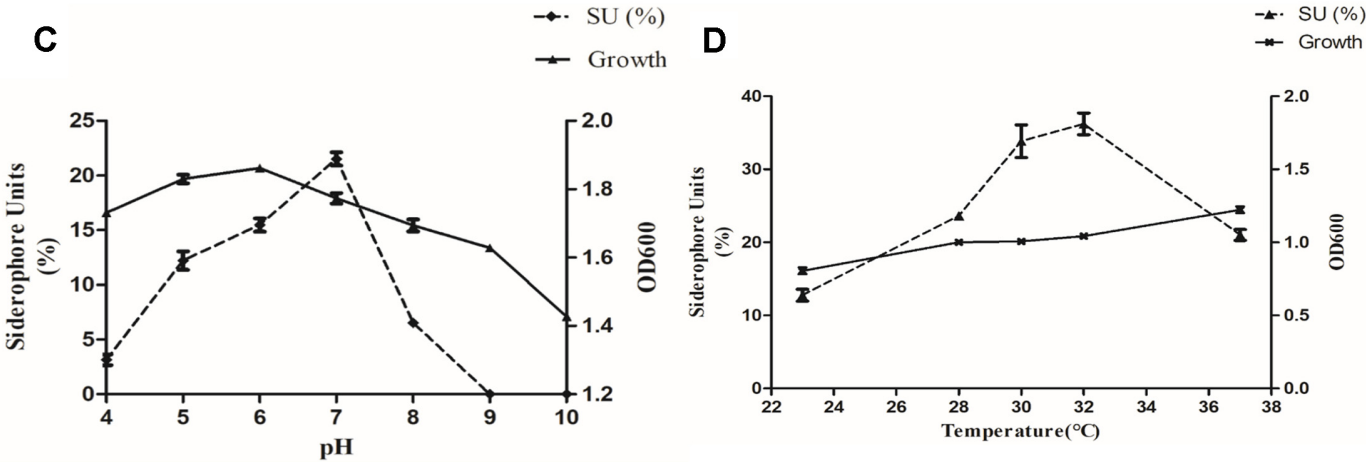

Fig. 1. Effect of fermentation time, pH, temperature on siderophore production and cell growth of GZDF3. (A) GZDF3 was cultured on SA-CAS agar plate, and the pink region means GZDF3 produces the siderophore. (B) The siderophore and cell growth curve graph. GZDF3 was cultured in SA medium and collected sample at different time points as shown in figure. OD600 was detected via multiscan spectrum instrument to evaluate the cell growth (right vertical axis). Siderophore content was determined by CAS liquid assay as described in Materials and Methods (left vertical axis). (C, D) Similar to (B), GZDF3 was cultured in SA medium with different $\mathrm{pH}(\mathbf{C})$, temperature $(\mathbf{D})$ as shown in figure. And sample was collected and detected as described above. All data were collected and analyzed in one graph. Error bars represent standard deviations of three replicates.

\section{Statistical Analysis}

Data were analyzed using SPSS 19.0 (SPSS Inc., USA) and expressed as the mean \pm standard error (SE). Statistical analyses were carried out using one-way analysis of variance. The statistical significance of the test was evaluated at the level of $p<0.05$ regarded as reliability.

\section{Results}

\section{Detection of Ferritin Synthesis by CAS Liquid and Plate Method}

After culturing B. brevis GZDF3 for $36 \mathrm{~h}$ in SA medium, $\mathrm{Fe}^{3+}$ in CAS-Fe $\mathrm{F}^{3+}$-HTDMA was transferred to ironfacilitated as the ability of siderophores to chelate iron ions stronger than that of CAS- $\mathrm{Fe}^{3+}$-HTDMA complex. As shown in Fig. 1A, a pale pink color change has appeared on the CAS blue solid agar of the SA-CAS plate. It has been demonstrated that the GZDF3 strain produced siderophores during growth on the surface (SA medium). The color change due to the production of siderophores was dynamically observed. The color reaction is consistent with the liquid detection, which further confirms that B. brevis GZDF3 can produce siderophores.

Secondly, different fermentation time points were chosen from 0 to $60 \mathrm{~h}$ with 6 -h intervals to investigate their effects on siderophore production and cell growth. As shown in Fig. 1B, the cell growth and siderophore production varied greatly with different fermentation times. The growth curve of GZDF3 was not a typical bacterial growth curve. The lag phase encompassed the initial $6 \mathrm{~h}$. In the logarithmic phase, from $6 \mathrm{~h}$ to $42 \mathrm{~h}$, cells kept growing (Fig. 1B, upper line). Thereafter, it entered the decline phase. The siderophore production trend was different from that of cell growth. We found that siderophores could be detected after $6 \mathrm{~h}$. In addition, the maximal production of siderophores was $29.67 \%$ measured at $48 \mathrm{~h}$. It is noteworthy that the siderophore production of

Table 1. Optimum conditions for synthesis of siderophore.

\begin{tabular}{lcccccc}
\hline & Carbon & Nitrogen & Temperature & Time & pH & SU \\
\hline SA pre-optimized & Sucrose $20 \mathrm{~g} / \mathrm{l}$ & Asparagine $2 \mathrm{~g} / \mathrm{l}$ & $28^{\circ} \mathrm{C}$ & $36 \mathrm{~h}$ & $\mathrm{pH}=7.6$ & $\mathbf{2 7 . 0 9 \%}$ \\
SA optimized & Sucrose $15 \mathrm{~g} / \mathrm{l}$ & Asparagine $2 \mathrm{~g} / \mathrm{l}$ & $32^{\circ} \mathrm{C}$ & $48 \mathrm{~h}$ & $\mathrm{pH}=7.0$ & $\mathbf{5 4 . 9 9 \%}$ \\
\hline
\end{tabular}


$25.76 \%$ reached another peak when the incubation time was $24 \mathrm{~h}$ (Fig. 1B, lower line). Thus, $48 \mathrm{~h}$ was chosen as the best fermentation time in the following experiments (Table 1).

pH of Medium. As shown in Fig. 1C, the siderophore production was influenced more obviously than the growth of GZDF3 by $\mathrm{pH}$ factor. The optimum $\mathrm{pH}$ for cell growth was 6.0. However, the highest siderophore production $(21.27 \% \mathrm{SU})$ was obtained at $\mathrm{pH} 7.0$. Therefore, the $\mathrm{pH}$ value was set to 7.0 in the following experiments for optimal siderophore production (Table 1).

Fermentation temperature. In our previous study, GZDF3 grew well at $37^{\circ} \mathrm{C}$ [19]. Taking soil temperature into account, the fermentation temperature was set from $23^{\circ} \mathrm{C}$ to $37^{\circ} \mathrm{C}$. The result showed that fermentation temperature not only affects the bacterial growth but also the siderophore production. In Fig. 1D, the highest yield of siderophore $(35.46 \% \mathrm{SU})$ occurred at $32^{\circ} \mathrm{C}$. However, the siderophore production decreased quickly as temperature increased above $32^{\circ} \mathrm{C}$. Interestingly, little change occurred in the GZDF3 cell growth as the temperature was increased. This indicated that fermentation temperature had little influence on cell growth. Hence, the temperature of $32^{\circ} \mathrm{C}$ was also used in the following experiments (Table 1).

\section{Effects of Media Composition on Siderophore Production and Cell Growth}

B. brevis GZDF3, belonging to Bacillus, was isolated from the rhizosphere soil of Pinellia ternata in Da Fang County, Guizhou Province, China. It has potential for use as biocontrol bacteria to cure pathogenic bacterial infection using $P$. ternata (an important herb in traditional Chinese medicine). Due to different microorganisms preferring different types and concentrations of media and fermentation conditions (temperature, time, etc.), we attempted to optimize these growth conditions for B. brevis GZDF3. As described in previously published papers, the culture medium for siderophore production of Bacillus sp. PZ-1 is modified SA medium [15, 17]. Using published papers on SA medium as reference $[32,33]$, we chose SA medium as the basic medium to optimize the siderophore production and growth of $B$. brevis GZDF3.

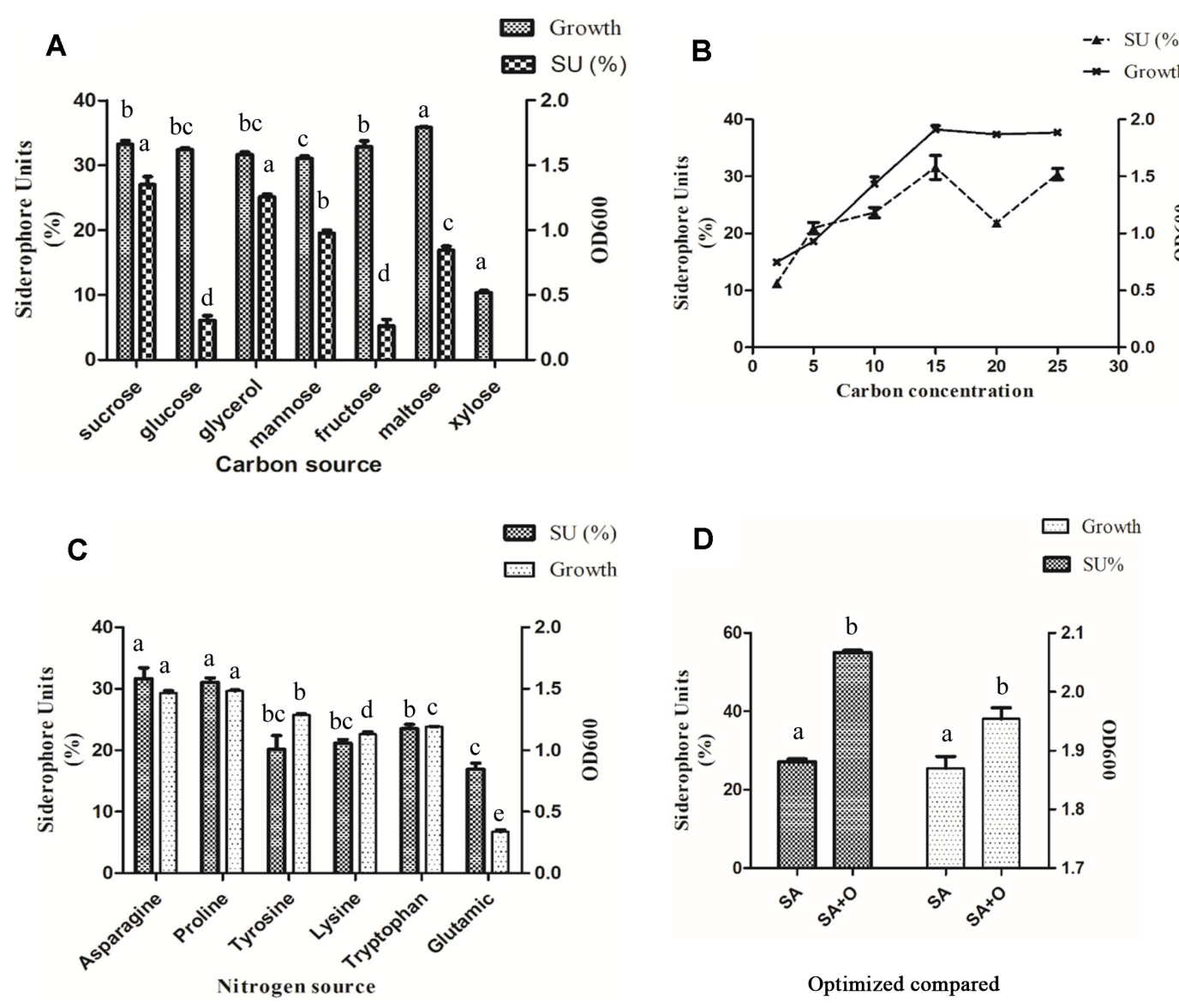

Fig. 2. Effect of carbon, nitrogen source on siderophore production and cell growth, and compared to the control under optimized culture conditions. GZDF3 was cultured on SA medium, with different carbon source (sucrose, glucose, glycerol, mannose, fructose, maltose, xylose), SA+O (optimized SA medium); (A) or different nitrogen source (Asparagine, Proline, Tyosine, Lysine, Tryptophan, Glutamic) (C) as shown in figure. (B) GZDF3 was cultured in SA medium with different concentrations sucrose as carbon source. (D) Changes in siderophore production and cell growth before and after optimization. And samples were collected and detected as described above. OD600 represent bacteria concentration of GZDF3 (right vertical axis) and SU means siderophore units (left vertical axis). Bars with the same letter are not significantly different $(p \geq 0.05)$ Error bars represent standard deviations of three replicates. 
In order to determine the optimal carbon source to induce higher siderophore production, carbon sources, including glycerol, monosaccharides (fructose, glucose, and mannose) and disaccharides (xylose, maltose, and sucrose) were tested. The cell growth and siderophore production were influenced by the carbon source. As shown in Fig. 2A, siderophore production varied greatly with carbon source, and the maximum production of siderophore $(27.02 \% \mathrm{SU})$ was obtained with sucrose. Glycerol ranked in second place, inducing high siderophore production $(25.10 \% \mathrm{SU})$, whereas glucose, mannose, fructose, and maltose were at $6.08 \%, 19.48 \%, 5.21 \%$, and $16.87 \%$, respectively. On the contrary, siderophore production couldn't be detected with xylose as the carbon source. It is obvious that maltose is the most favorable carbon source for GZDF3 growth, whereas xylose is the most unfavorable in comparison with the other options.

The concentration of sucrose was optimized, as shown in Fig. 2B. It was observed that when the sucrose concentration was $15 \mathrm{~g} / \mathrm{l}$, the highest siderophore production reached $31.59 \%$. Therefore, the following experiments were all performed with $15 \mathrm{~g} / \mathrm{l}$ sucrose as the carbon source (Table 1 ).

As shown in Fig. 2C, various amino acids showed different effects on siderophore production. Asparagine induced the highest siderophore yield of $31.64 \%$, and the minimum production of siderophore at $16.92 \%$ was obtained with the addition of glutamic acid. Therefore, asparagine was used as nitrogen source in the following experiments (Table 1). As shown in Fig. 2D and Table 1, the optimized SA medium (SA+O) significantly increased the production of siderophores, from $27.09 \%$ to $54.99 \%$, and also promoted cell growth in comparison with the basic SA medium.

Effects of Metal Ions on Siderophore Production and Cell Growth

Due to the influence of metal ions on siderophore production $[19,21]$, four metal ions $\left(\mathrm{Fe}^{3+}, \mathrm{Mn}^{2+}, \mathrm{Cu}^{2+}, \mathrm{Zn}^{2+}\right)$ were investigated for their effects on siderophore synthesis in this study.

As shown in Fig. 3, different concentrations of ferric ion $(0,5,10,50,100,200,400$, and $800 \mu \mathrm{mol} / \mathrm{l})$ of $\mathrm{FeCl}_{3}$ were added to the SA medium to assess their function on siderophore production. The increase of $\mathrm{Fe}^{3+}$ concentrations had a negative effect on siderophore production, especially when the concentration was above $10 \mu \mathrm{mol} / \mathrm{l}$. A significant reduction trend of siderophore production was found as $\mathrm{FeCl}_{3}$ concentration was increased. It is interesting to note that the highest siderophore production was without $\mathrm{Fe}^{3+}$ medium. $\mathrm{As} \mathrm{FeCl}_{3}$ concentration increased from 1 to $5 \mu \mathrm{mol} / \mathrm{l}$, the amount of produced siderophore decreased by $80 \%$. This production was almost completely suppressed when $\mathrm{FeCl}_{3}$ was at $10 \mu \mathrm{mol} / \mathrm{l}$. Meanwhile, increased cell growth was observed as the $\mathrm{FeCl}_{3}$ concentration increased from 0 to $10 \mu \mathrm{mol} / \mathrm{l}$. However, the cell growth was slightly repressed when $\mathrm{FeCl}_{3}$
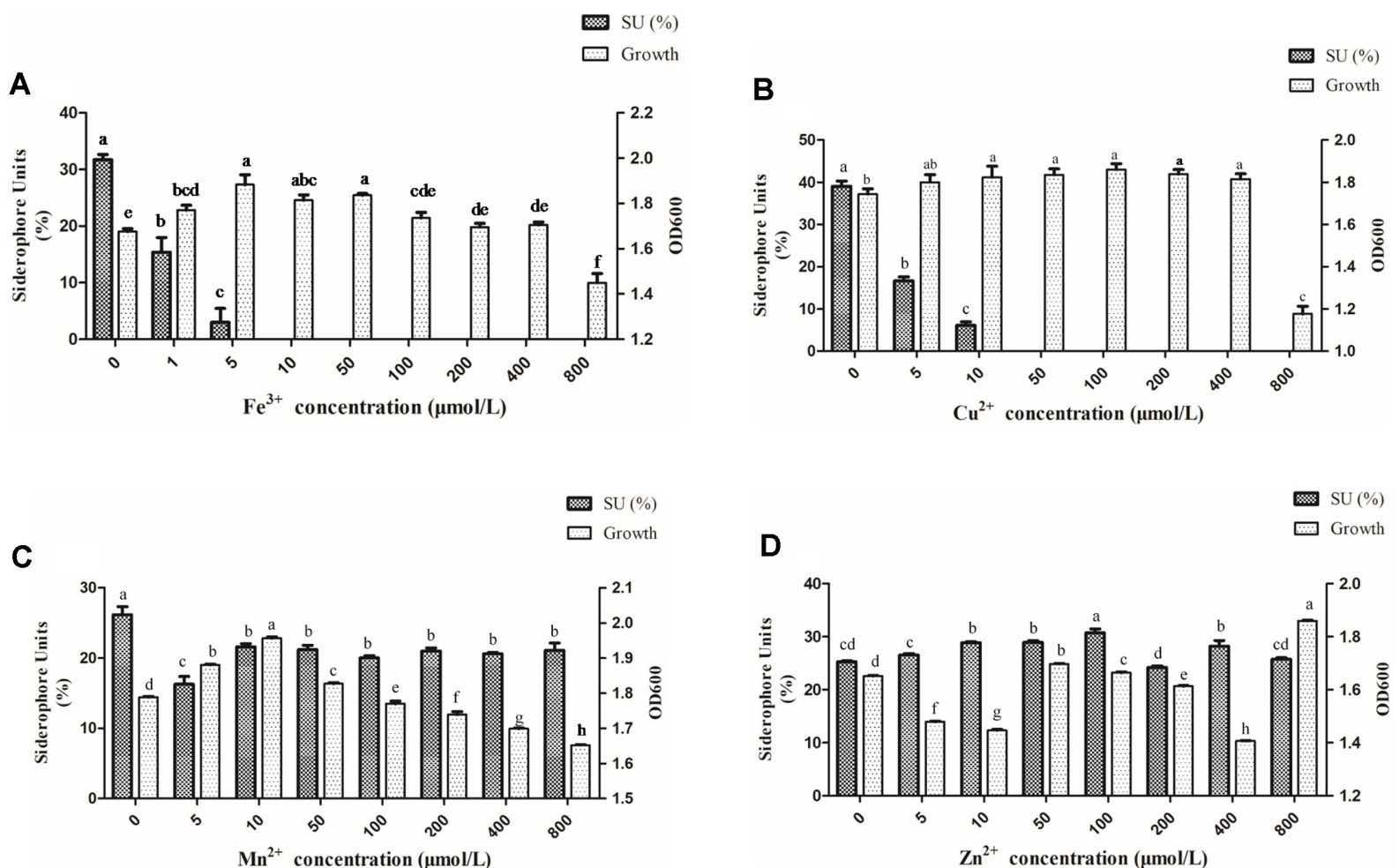

Fig. 3. Effect of metal ions on siderophore and bacterial growth. Similar to Fig. 2, GZDF3 was cultured in SA medium with different concentrations of metal iron $\left(\mathrm{Fe}^{3+}, \mathrm{Mn}^{2+}, \mathrm{Cu}^{2+}, \mathrm{Zn}^{2+}\right)$ as shown in figure. And sample was collected and detected as described above. OD600 represent bacteria concentration of GZDF3 (right vertical axis) and SU means siderophore units (left vertical axis). Error bars represent standard deviations of three replicates. Bars with the same letter are not significantly different $(p \geq 0.05)$ Error bars represent standard deviations of three replicates. 
concentration was higher than $10 \mu \mathrm{mol} / \mathrm{l}$ (Fig. 3A).

Similar to Fe ion, the siderophore production was also suppressed by the increased $\mathrm{Cu}^{2+}$ concentration. There was nearly no siderophore production at the concentration of $50 \mu \mathrm{mol} / \mathrm{l}$ (Fig. 3B). At the same time, no significant change was observed for the cell growth from 0 to $400 \mu \mathrm{mol} / 1$. But the cell growth was decreased with $800 \mu \mathrm{mol} / \mathrm{l}$ of $\mathrm{Cu}^{2+}$ (Fig. 3B). This may because there is a tolerance range of GZDF3 growth to the concentration of $\mathrm{Cu}^{2+}$. If it is within a certain range, the growth won't change too much. If the concentration of $\mathrm{Cu}^{2+}$ changes very much, this will be reflected in the growth of GZDF3.

The amount of siderophores decreased as the concentration of $\mathrm{Mn}^{2+}$ increased in this study, especially at the concentration of $5 \mu \mathrm{mol} / \mathrm{l}$ (Fig. 3C). Interestingly, the $\mathrm{Mn}^{2+}$ was observed to stimulate siderophore production and cell growth with the concentration from 5 to $50 \mu \mathrm{mol} / \mathrm{l}$ as shown in Fig. 3C. No significant change in the siderophore production was observed with $\mathrm{Mn}^{2+}$ concentration increasing from 100 to $800 \mu \mathrm{mol} / \mathrm{l}$. Meanwhile, the cell growth was remarkably decreased (Fig. 3C).

As shown in Fig. 3D, it is interesting to find that $\mathrm{Zn}^{2+}$ had a relatively weak effect on siderophore production in comparison with the other metals. There was only a slight increase in siderophore production with increasing $\mathrm{Zn}^{2+}$. The cell growth was inhibited by increasing $\mathrm{Zn}^{2+}$ from 0 to $10 \mu \mathrm{mol} / \mathrm{l}$. However, the inhibition effect did not show a clear trend with the increase of $\mathrm{Zn}^{2+}$, especially from 50 to $200 \mu \mathrm{mol} / \mathrm{l}$.

These results indicated that the effect of metal ions on siderophore production of GZDF3 varied with metal species and concentration. It would not benefit siderphore production of GZDF3 to add metal ions $\left(\mathrm{Fe}^{3+}, \mathrm{Cu}^{2+}\right.$, $\left.\mathrm{Mn}^{2+}, \mathrm{Zn}^{2+}\right)$ in the fermentation process.

\section{Antifungal Activity of Crude Siderophore Solution}

As shown in Table 2, C. albicans growth was inhibited by GZDF3 fermentation solution which contained siderophore. The inhibition zones of GZDF3 fermentation broth with SA medium and optimized SA medium were $41 \pm 3 \mathrm{~mm}$ and $45 \pm 3 \mathrm{~mm}$, respectively. In order to investigate the dose inhibition effect of siderophore to fungal growth, $1 \mathrm{mg} / \mathrm{ml}, 5 \mathrm{mg} / \mathrm{ml}$, and $10 \mathrm{mg} / \mathrm{ml} \mathrm{Fe}$ (III)-IMAC column-purified siderophore solutions were added to the hole in the plate. Ketoconazole at $1 \mathrm{mg} / \mathrm{ml}, 5 \mathrm{mg} / \mathrm{ml}$, and $10 \mathrm{mg} / \mathrm{ml}$ was used as positive control, while $1 \mathrm{mg} / \mathrm{ml}, 5 \mathrm{mg} / \mathrm{ml}$, and $10 \mathrm{mg} / \mathrm{ml}$ of $\mathrm{Fe}^{3+}$-treated fermented broth were used as complementary comparison experiment control. As showed in Table 2, both inhibition zones of purified siderophore and ketoconazole become wider as the dose was increased. On the other hand, only ketoconazole was observed with a small inhibition zone of $12 \pm 3 \mathrm{~mm}$. However, purified siderophore has a wider inhibition zone relative to ketoconazole. Interestingly, the $\mathrm{Fe}^{3+}$-treated fermentation medium showed decline of the inhibition zone. This is consistent with the inhibition effect of Fe (III)-IMAC column-purified siderophore to C.albicans. When we use the Fe(III)-IMAC column to purify siderophore, the siderophore concentration is higher in the solution. However, $\mathrm{Fe}^{3+}$ treatment inhibits the siderophore production in the fermentation medium. Thus, the inhibition effect to C.albicans was attenuated. According to the results in Table 1, strain GZDF3 is highly antagonistic to the pathogen C. albicans. Also, siderophore plays an important role in this effect.

In order to further investigate the antifungal effect of GZDF3 siderophore to C. albicans, the time-kill curve was drawn with different concentrations of siderophores and different time points. Here, 2,500, 1,250, 625, and $312.5 \mu \mathrm{g} / \mathrm{ml}$ of purified siderophores all showed strong inhibitory effect to C. albicans throughout the whole period (Fig. 4). Moreover, the inhibition effect is decreased as the concentration reduces. Meanwhile, all of the four concentrations of siderophores have obvious inhibitory effect from $6 \mathrm{~h}$ to $12 \mathrm{~h}$. These results show that the siderophores have significant effect during the 6th to 12th hour (Fig. 4) and also that purified siderophore has a significant antagonistic effect on C. albicans.

Siderophore produced by GZDF3 has a stronger antifungal effect than ketoconazole. Siderophore regulates intracellular ferrous ion for survival whereas ketoconazole and amphotericin B inhibit levels of ergosterol, which is one of the components in the membrane. The elucidation of relationship (synergistic or additive effect) between siderophore and antibiotics would be quite useful information. Due to ketoconazole having been reported for drug resistance phenomenon in clinical use, the synergistic or additive effect experiments were carried out with siderophore and amphotericin B in subsequent experiments.

As shown in Table 3, both siderophore and amphotericin B have been associated with great decline in MIC concentration when these two are combined together in comparison with being used alone. Also, the FIC value is

Table 2. Antifungal activity of GZDF3 against Candida albicans.

\begin{tabular}{lc}
\hline \multicolumn{1}{c}{ Candida albicans } & Zone of inhibition $(\mathrm{mm})$ \\
\hline SA medium pre-optimized & $41 \pm 3$ \\
SA medium optimized & $45 \pm 3$ \\
$1 \mathrm{mg} / \mathrm{ml} \mathrm{ketoconazole}$ & $12 \pm 3$ \\
$1 \mathrm{mg} / \mathrm{ml}$ purified siderophore & $(-)$ \\
$1 \mathrm{mg} / \mathrm{ml} \mathrm{Fe}{ }^{3+}$ medium & $(-)$ \\
$5 \mathrm{mg} / \mathrm{ml} \mathrm{ketoconazole}$ & $20 \pm 3$ \\
$5 \mathrm{mg} / \mathrm{ml}$ purified siderophore & $30 \pm 3$ \\
$5 \mathrm{mg} / \mathrm{ml} \mathrm{Fe}{ }^{3+}$ medium & $19 \pm 3$ \\
$10 \mathrm{mg} / \mathrm{ml} \mathrm{ketoconazole}$ & $30 \pm 3$ \\
$10 \mathrm{mg} / \mathrm{ml}$ purified siderophore & $38 \pm 3$ \\
$10 \mathrm{mg} / \mathrm{ml} \mathrm{Fe}{ }^{3+}$ medium & $20 \pm 3$ \\
\hline
\end{tabular}




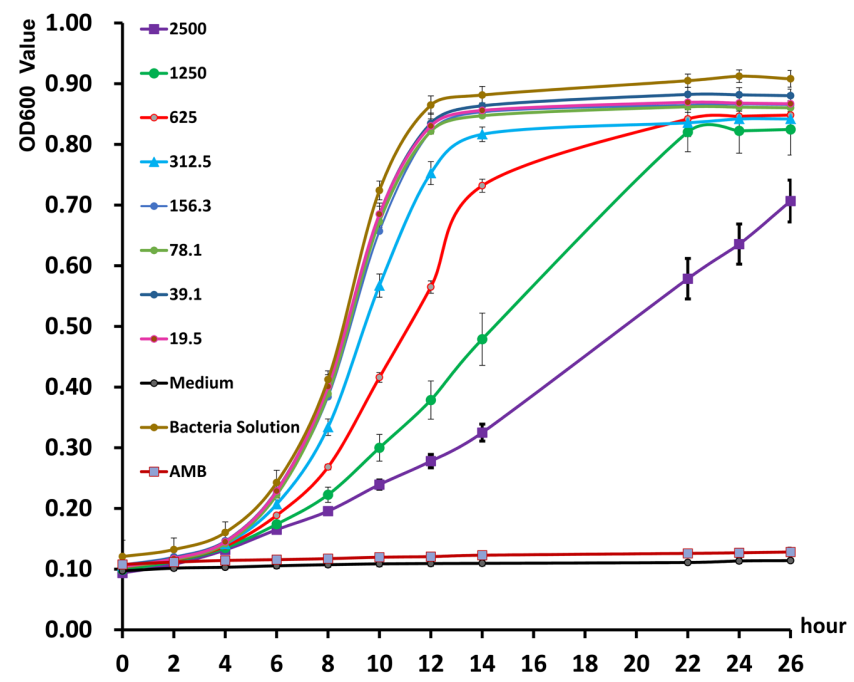

Fig. 4. Time-kill curves of Bacillus brevis GZDF3 siderophore to C. albicans. C. albicans were cultured in the potato dextrose broth (PDB) medium. Different concentrations siderphore which was purified by Fe(III)-IMAC column were add to the medium according to the figure. PDB medium-only group (Medium) used as negative control. C. albicans with PDB medium (Bacteria Solution) is control group. And the amphotericin B (AMB) used as posititive control according to the figure. Then samples were detected by at different time points as described in the figure. OD600 value represents bacteria concentration of C. albicans. Error bars represent standard deviations of three replicates.

0.031 , which is less than 0.5 . It has been shown that siderophore produced by GZDF3 could have a synergistic effect on C. albicans when combined with amphotericin B.

\section{Discussion}

In this study, we characterized the siderophore production of B. brevis GZDF3 (Fig. 1). The optimal fermentation conditions of siderophore production by B. brevis GZDF3 were reported as shown in Fig. 2 and Table 1. In addition, adding metal ions $\left(\mathrm{Fe}^{3+}, \mathrm{Cu}^{2+}, \mathrm{Mn}^{2+}, \mathrm{Zn}^{2+}\right)$ in the fermentation process inhibited the siderophore production of GZDF3 (Fig. 3). Moreover, Fe(III)-IMAC column-purified siderophores of GZDF3 have obvious great anti-fungal activity to C. albicans (Fig. 4 and Table 2). In addition, GZDF3 siderophore possesses a synergistic resistance effect with amphotericin B to C. albicans (Table 3).

Culture medium and various abiotic factors could influence the siderophore production. The degree of acidity or alkalinity $(\mathrm{pH})$ is a significant factor in siderophore production by $P$. aeruginosa PSS [34]. The maximum siderophore production was obtained in Pseudomonas, such as Pseudomonas syringae BAF.1, Pseudomonas aeruginosa and fluorescent pseudomonads, with medium of $\mathrm{pH} 7.0[16,17,34]$. However, $\mathrm{pH} 6.0$ also proved suitable for producing hydroxamate siderophore in marine Pseudomonas aeruginosa and Bacillus sp. PZ-1 [15, 17, 35]. The maximum siderophore production (SU) of B. brevis GZDF3 was obtained at $\mathrm{pH}$ 7.0, which is similar to the conclusions drawn above. To sum up, a neutral $\mathrm{pH}$ is likely suitable for siderophore production of microbes, and our results show the same (Fig. 1 and Table 1).

Pyoverdine levels were much higher at $15^{\circ} \mathrm{C}$ to $20^{\circ} \mathrm{C}$, but maximum cell growth was between $20^{\circ} \mathrm{C}$ and $30^{\circ} \mathrm{C}$ in Pseudomonas fluorescens [16]. In Pseudomonas aeruginosa, maximum siderophore production was recorded at $30^{\circ} \mathrm{C}$. These were similar in GZDF3 for maximum siderophore yields at $30^{\circ} \mathrm{C}$ to $32^{\circ} \mathrm{C}$ in our study (Fig. $1 \mathrm{~B}$ ). It was reported that the siderophore production reached the peak when the fermentation time was $24 \mathrm{~h}$ for incubation of Alcaligenes faecalis BCCM ID 2374 [36, 37]. It was shown that the maximum siderophore production by Bacillus megaterium was obtained in the stationary phase [38]. Interestingly, the optimal fermentation time for siderophore production of GZDF3 has two peaks $(24 \mathrm{~h}, 48 \mathrm{~h})$ in our study. The first one is at $24 \mathrm{~h}$ and is weaker than the one at $48 \mathrm{~h}$. Meanwhile, the cell growth of GZDF3 increases from $24 \mathrm{~h}$ to $48 \mathrm{~h}$. Thus, inoculation of GZDF3 for $48 \mathrm{~h}$ in stationary phase is a good choice, all things considered.

Carbon source and nitrogen source determined the metabolism of microbes and also the quality of medium.

Table 3. Synergistic MIC value and FIC index of each drug when combined.

\begin{tabular}{lccc}
\hline & \multicolumn{2}{c}{ MIC } & FIC \\
\cline { 2 - 4 } & Combine & Alone & 0.031 \\
\hline Siderophore & 64 & 1020 & 19.5 \\
Amphotericin B & 0.781 & 19.5 & 0 \\
\hline
\end{tabular}

Note: FIC index $<0.5$, Synergistic effect 
Carbon sources such as sucrose and glycerol are important factors in cell growth and siderophore production of microbes. It is good for siderophore production in Streptomyces fulvissimus when sucrose is used as carbon source [13]. However, another report found that the highest siderophore production was detected with glycerols as carbon source in Bacillus megaterium [14]. Glucose was also found to influence the siderophore production in P. fluorescens CHA [39]. In Pseudomonas syringae BAF.1 and Bacillus sp. PZ-1, glucose was also the suitable carbon source to induce siderophore production $[15,17]$. Herein, sucrose was proved to be the best carbon source to stimulate siderophore production in SA medium in our study (Fig. 2A).

Many reports found that culture media containing asparagine showed highly effective siderophore production by Pseudomonas syringae and Pseudomonas viridiflava LMG2352 strains [40]. We also found that asparagine and proline are suitable for inducing siderophore production and cell growth of GZDF3. However, the SU is higher in GZDF3 with asparagine as nitrogen source than proline. Thus, these results are mainly in accordance with those reported in Pseudomonads [35, 39, 40].

Metal ions have been reported to regulate siderophore production of microbes in the environment [41, 42]. In the present study, four metal ions $\left(\mathrm{Fe}^{3+}, \mathrm{Cu}^{2+}, \mathrm{Mn}^{2+}, \mathrm{Zn}^{2+}\right)$ were selected to evaluate the effect on siderophore production of GZDF3. Surprisingly, the production of siderophore was suppressed by $\mathrm{Fe}^{3+}$ (Fig. 3). When the concentration of Fe ion reached to $10 \mu \mathrm{mol} / \mathrm{L}$, siderophore could not be detected. This result was similar to that of Pseudomonas syringae BAF.1 [17]. However, it was found that the threshold level of $\mathrm{Fe}^{3+}$ suppressing siderophore production in gram-positive isolates was more than $30 \mu \mathrm{mol} / \mathrm{l}[16]$.

As shown in Fig. 3A, siderophore production is decreased in a $\mathrm{Fe}^{3+}$ concentration-dependent manner. The inhibition zone of $1 \mathrm{mg} / \mathrm{ml} \mathrm{Fe}^{3+}$-treated GZDF3 fermentation medium is undetected. Also, as the concentration of $\mathrm{Fe}^{3+}$ increased to $5 \mathrm{mg} / \mathrm{ml}$, the inhibition zone also increased to $19 \pm 3 \mathrm{~mm}$. However, the inhibition zone only changed to $20 \pm 3 \mathrm{~mm}$ when the concentration of $\mathrm{Fe}^{3+}$ changed to $10 \mathrm{mg} / \mathrm{ml}$. On the other hand, the inhibition zones of $10 \mathrm{mg} / \mathrm{ml}$ of purified siderophore or ketoconazole are $38 \pm 3$ or $30 \pm 3 \mathrm{~mm}$, respectively. These data show that the size of the inhibition zone is not increased in a typical $\mathrm{Fe}^{3+}$ concentration-dependent manner totally in Table 2. As $\mathrm{Fe}^{3+}$ concentration increased from 0 to $1 \mathrm{mg} / \mathrm{ml}$, the siderophore production nearly decreased by half. So, the antifungal effect attenuated greatly, while the inhibition zone could not be detected at the same time. In addition, as the concentration of $\mathrm{Fe}^{3+}$ increased to $5 \mathrm{mg} / \mathrm{ml}$, the inhibition zone appeared and didn't change with further increase of the $\mathrm{Fe}^{3+}$. This indicated to us that there is probably some antifungal substance produced by GZDF3 with the stimulation of low-dose $\mathrm{Fe}^{3+}(5 \mathrm{mg} / \mathrm{ml})$. However, the substance didn't increase as the $\mathrm{Fe}^{3+}$ concentration increased $(10 \mathrm{mg} / \mathrm{ml}$ ). Thus, the inhibition zone didn't change much (from 19 to $20 \mathrm{~mm}$ ). Also, as shown in Fig. 3, Table 2, the optimized SA medium and $10 \mathrm{mg} / \mathrm{ml}$ of purified siderophore have great antifungal effect with inhibition zones of $45 \pm 3$ or $38 \pm 3 \mathrm{~mm}$. These results showed siderophore is one of the key components of fermentation medium produced by GZDF3 and plays an important role in resistance to C.albicans.

It was reported that $\mathrm{Al}^{3+}, \mathrm{Cd}^{2+}, \mathrm{Cu}^{2+}$, and $\mathrm{Ni}^{2+}$ stimulated siderophore production by Streptomyces sp. [19]. One $\mu \mathrm{M}$ concentration of $\mathrm{CuCl}_{2}, \mathrm{MgCl}_{2}$, and $\mathrm{ZnSO}_{4}$ resulted in optimal siderophore yield in Alcaligenes faecalis $[33,34]$. In contrast, we discovered that $\mathrm{Cu}^{2+}$ has the same effect on siderophore production as $\mathrm{Fe}^{3+}$. The higher the amount of $\mathrm{Cu}^{2+}$ added, the lower the amount of siderophore that was produced. The threshold level of $\mathrm{Cu}^{2+}$ that inhibited siderophore production in GZDF3 was above $50 \mu \mathrm{mol} / \mathrm{l}$ (Fig. 3).

It has been reported that the inhibition of siderophore synthesis in zotobacter vinelandii was found at elevated levels of $\mathrm{Mn}^{2+}$ and $\mathrm{Zn}^{2+}$ [37]. Surprisingly, in this study, $\mathrm{Mn}^{2+}$ was found to increase growth of GZDF3 with the concentration increased from 0 to $50 \mu \mathrm{mol} / \mathrm{l}$, while the siderophore production decreased (Fig. 3C).

Reports show that high concentrations of $\mathrm{Zn}^{2+}$ ion increased the production of siderophore in iron-limited cultures in fungi and bacteria $[20,21]$. However, no significant trend was found in siderophore production and cell growth by GZDF3 with the increase of $\mathrm{Zn}^{2+}$ in comparison with the other metals in our study (Fig. 3). These results suggested that the effect of metal on siderophore production varied with metal species and concentrations of GZDF3. $\mathrm{Fe}^{3+}$ and $\mathrm{Cu}^{2+}$ could strongly inhibit the siderophore production of GZDF3 as the increase in medium, and $\mathrm{Mn}^{2+}, \mathrm{Zn}^{2+}$ have weaker effects (Fig. 3).

Siderophore produced by Alcaligenes feacalis showed suppressive activity against F. oxysporum NCIM1008 [36]. Many reports suggested that siderophores produced by P. fluorescens MPF47 have strong biocontrol abilities against R. solani [43]. Moreover, it was also discovered that siderophore produced by Seudomonas syringae BAF. 1 exhibited prominent antagonistic activity against Fusarium oxysporum in the absence of $\mathrm{FeCl}_{3} \cdot 6 \mathrm{H}_{2} \mathrm{O}$ [17]. Interestingly, siderophore named Bacillibactin produced by SQR9 was up-regulated when it was confronted with fungi [44]. It was also shown that siderophores produced by Pseudomonas fluorescens BBc6R8 inhibited the growth of the actinomycete Streptomyces ambofaciens ATCC23877 [45, 46]. Compared with purified siderophores, cell-free supernatant of $P$. putida had a greater antagonistic activity towards fungi [47]. All of this reminds us that siderophores have anti-fungal activity $[45,48]$. Yasmin reported the importance of siderophore and ergosterol biosynthetic pathways for fungal virulence and antifungal treatment, as iron starvation down-regulates the cellular ergosterol content but up-regulates siderophore (TAFC) production by Aspergillus fumigatus [26]. In our research, both crude and Fe(III)-IMAC column-purified siderophore solution produced by GZDF3 showed prominent antagonistic activity to C. albicans (Fig. 4 and Table 2). This should be useful in the prevention and control of fungus pathogens.

Siderophore regulates intracellular ferrous ion for survival, whereas ketoconazole inhibits ergosterol, which is one of the components in the membrane. The action mode of amphotericin B leads to pore formation on the membrane by binding to ergosterol, thereby leaking intracellular essential components. The elucidation of the relationship (synergistic or additive effect) is interesting. Iron is an essential nutrient for all living cells. There is increasing evidence that interfering with the iron homeostasis of $C$. albicans can improve its antifungal response. 
In line with this, our results show that siderophore produced by GZDF3 could act synergistically with amphotericin B when used against C. albicans.

In conclusion, we optimized the fermentation conditions of siderophore production by GZDF3, and characterized the siderophore's antifungal function against $C$. albicans. These results could provide a useful reference for understanding the antifungal activity of GZDF3 to other fungal pathogens and its potential application in the cultivation of Pinellia ternata. However, further study is required to explore the exact antagonistic mechanism of siderophore produced by GZDF3 to C. albicans in the future.

\section{Acknowledgments}

This work was supported by grants from the Medical Biotechnology Engineering Research Center of Guizhou Medical University (2016002), Guizhou Science and Technology Plan Project (Guizhou Science and Technology Support Program [2017]2833) and The Academic New Seedling Cultivation and Innovation Exploration Project (Guizhou Science and Technology platform talents [2018]5779-53), United Foundation of Collaboration Scientific Project between the Bureau of Science \& Technology of Guiyang City and Guizhou Medical University (ZHUKEJ [20151001] SHE-15); Doctoral Cultivating Fund of Guizhou Medical University (Academy-PHD-J2014-018), scientific plan of Guizhou provincial health and fitness commission (gzwjkj2015-1-028). We also thank Dr. Emon Sultan Ahmed at Karolinska Institute for revising this manuscript.

\section{Conflict of Interest}

The authors have no financial conflicts of interest to declare.

\section{Reference}

1. Gregory JA, David MF 2017. Current understanding of iron homeosisi. Am. J. Clin. Nutr. 106: 1559S-1566S.

2. Simon CA, Andrea KR, Franciso RQ. 2003. Bacterial Iron Homeostasisc. FEMS Microbiol. Rev. 27: 215-237.

3. Arora NK, Verma M. 2017. Modified microplate method for rapid and efficient estimation of siderophore produced by bacteria. 3Biotech 7: 381.

4. Ringo S, Marlene KA, Katarzyna S, Oliver W, Drik T. 2018. Analysis of desferrioxamine-like siderophores and their capability to selectively bind metals and metalloids: development of a robust analytical RP-HPLC method. Res. Microbiol. 169: 598-607.

5. Albelda BM, Monachon M, Joseph E. 2019. Siderophores: from natural rolesto potential applications. Adv. Appl. Microbiol. 106: 193225 .

6. De Serrano LO, Camper AK, Richards AM. 2016. An overview of siderophores for iron acquisition in microorganisms living in the extreme. Biometals 29: 551-571.

7. Wilson BR, Bogdan AR, Miyazawa M, Hashimoto K, Tsuji Y. 2016. Siderophores in iron metabolism: from mechanism to therapy potential. Trends Mol. Med. 22: 1077-1090.

8. Levent hal GE, Ackermann M, Schiessl KT. 2019. Why microbes secrete molecules to modify their environment: the case of ironchelating siderophores. J. R. Soc. Interface 16: 1-13.

9. Shi JL, Li YQ, Hu KM, Ren JG, Liu HM. 2015. Isolation and identification of pathogens from rotted root of Pinellia ternata in Guizhou province. Microbiol. China 42: 289-299.

10. Ahmed E, Holmstrom SJM. 2014. Siderophores in environmental research: roles and applications. Microb. Biotechnol. 7: 196-208.

11. Qi B, Han M. 2018. Microbial siderophore enterobactin promotes mitochondrial iron uptake and development of the host via interaction with ATP synthase. Cell 175: 571-582.

12. Sheng MM, Jia HK, Tao XM, Zeng LN, Zhang TT, Hu Z Q, et al. 2018. Mining, isolation and identification of siderophore synthesis gene from Brevibacillus brevis GZDF3. Am. J. Biochem. Biotechnol. 14: 200-209.

13. Bendale MS, Chaudhari BL, Chincholkar SB. 2009. Influence of environmental factors on siderophore production by Streptomyces fulvissimus ATCC 27431. Curr. Trends Biotechnol. Pharm. 3: 362-371.

14. Santos S, Neto IF, Machado MD, Soares HMVM, Soares EV. 2014. Siderophore production by Bacillus megaterium: effect of growth phase and cultural conditions. Appl. Biochem. Biotechnol. 172: 549-560.

15. Yu S, Teng C, Bai X, Liang JS, Song T, Dong LY, et al. 2017. Optimization of siderophore production by Bacillus sp. PZ-1 and its potential enhancement of phytoextration of PB from soil. J. Microbiol. Biotechnol. 27: 1500-1512.

16. Sayyed RZ, Badgujar MD, Sonawane HM, Mhaske MM, Chincholkar SB. 2005. Production of microbial iron chelators (siderophores) by fluorescent pseudomonads. Ind. J. Biotechnol. 4: 484-490.

17. Yu SM, Teng CY, Bai X, Liang JS, Song T, Dong LY, et al. 2017. Characterization of siderophore produced by Pseudomonas syringae BAF.1 and its inhibitory effects on spore germination and mycelium morphology of Fusarium oxysporum. J. Microbiol. 55: 877-884.

18. Waldron KJ, Tottey S, Yanagisawa S, Dennison C, Robinson NJ. 2007. A periplasmic iron-binding protein contributes toward inward copper supply. J. Biol. Chem. 282: 3837-3846.

19. Dimkpa CO, Merten D, Svatos A, Buchel G, Kothe E. 2009. Metal-induced oxidative stress impacting plant growth in contaminated soil is alleviated by microbial siderophores. Soil Biol. Biochem. 41: 154-162.

20. Khalid AH, Jin HJ. 2019. Zinc Ions Affect Siderophore Production by Fungi Isolated from the Panax ginseng Rhizosphere. J. Microbiol. Biotechnol. 29: 105-113.

21. Gaonkar T, Bhosle S. 2013. Effect of metals on a siderophore producing bacterial isolate and its implications on microbial assisted bioremediation of metal contaminated soils. Chemosphere 93: 1835-1843.

22. Naik MM, Dubey SK. 2011. Lead-enhanced siderophore production and alteration in cell morphology in a Pb-resistant Pseudomonas aeruginosa strain 4EA. Curr. Microbiol. 62: 409-414.

23. Nobile CJ, Johnson AD. 2015. Candida albicans biofilms and human disease. Annu. Rev. Microbiol. 69: 71-92.

24. Lv QZ, Yan L, Jiang YY. 2016. The synthesis, regulation, and functions of sterols in Candida albicans: well-known but still lots to learn. Virulence 7: 649-659.

25. Jakub S, Jakub M, Przemysław B, Anna K. 2019. A Crucial role for ergosterol in plasma membrane composition, localisation, and activity of Cdrlp and H+-ATPase in Candida albicans. Microorganisms 7: 378.

26. Yasmin S, Alcazar FL, Gründlinger M, Puempel T, Cairns T, Blatzer M, 2012. Mevalonate governs interdependency of ergosterol and siderophore biosyntheses in the fungal pathogen Aspergillus fumigatus. Proc. Natl. Acad. Sci. USA 109: 497-504.

27. Savage KA, Parquet MC, Allan DS, Davidson RJ, Holbein BE, Lilly EA,.2018. Iron restriction to clinical isolates of Candida albicans by the novel chelator DIBI inhibits growth and increases sensitivity to azoles in vitro and in vivo in a murine model of experimental vaginitis. Antimicrob. Agents Chemother. 62: e02576-17. 
28. Schwyn, B. and Neilands, JB. 1987. Universal chemical assay for the detection and determination of siderophore. Anal. Biochem. 160: 47-56.

29. Li YY, Jiang W, Gao RJ, Cai YJ, Guan ZB, Liao XR. 2018. Fe(III)-based immobilized metal-affinity chromatography (IMAC) method for the separation of the catechol siderophore from Bacillus tequilensis CD36. 3 Biotech. 8: 31-36.

30. Ierusalimskii ND, Konova IV, Neronova NM. 1956. Determination of vitamins and antibiotics by diffusion in agar. I. Simplified computations for the cup method. Mikrobiologiia 28: 433-443.

31. Díaz VM, Villa P, Frías A. 2002. Evaluation of the siderophores production by Pseudomonas aeruginosa PSS. Rev. Latinoam Microbiol. 44: 112-117.

32. Scher Fran M, Baker Ralph. 1982. Effect of Pseudomonas putida and a synthetic iron chelator on induction of soil suppressiveness to Fusarium wilt pathogens. J. Phytopathol. 72: 1567-1573.

33. Manninen Merja, Mattila-Sandholm Tiina. 1994. Methods for the detection of Pseudomonas siderophores. J. Microbiol. Method 19: 223-234.

34. Le VH, Olivera C, Spagnuolo J, Davies IG, Rakonjac J. 2020. In vitro synergy between sodium deoxycholate and furazolidone against enterobacteria. BMC Microbiol. 20(1): 5.

35. Manwar AV, Khandelwal SR, Chaudhari BL, Meyer JM, Chincholkar SB . 2004. Siderophore production by a marine Pseudomonas aeruginosa and its antagonistic action against phytopathogenic fungi. Appl. Biochem. Biotechnol. 118: 243-251.

36. Sayyed RZ, and Chincholkar SB. 2009. Siderophore producing Alcaligenes feacalis: more biocontrol potential visavis chemical fungicide. Curr. Microbiol. 58: 47-51.

37. Sayyed RZ, Chincholkar SB. 2010. Growth and siderophores production in Alcaligenes faecalis is regulated by metal ions. Indian J. Microbiol. 50: 179-182.

38. Shaikh SS, Wani SJ, Sayyed RZ. 2016. Statistical-based optimization and scale-up of siderophore production process on laboratory bioreactor. 3 Biotech. 6(1): 69.

39. Duffy BK, Défago G. 2000. Controlling instability in gacS-gacA regulatory genes during inoculant production of Pseudomonas fluorescens biocontrol strains. Appl. Environ. Microbiol. 66: 3142-3150.

40. Bultreys A, Gheysen L. 2000. Production and comparison of peptide siderophores from strains of distantly related pathovars of Pseudomonas syringae and Pseudomonas viridiflava LMG2352. Appl. Environ. Microbiol. 66: 325-331.

41. Johnstone TC, Nolan EM. 2015. Beyond iron: non-classical biological functions of bacterial Siderophores. Dalton Trans. 44: 63206339.

42. Wang Z, Schenkeveld WD, Kraemer SM, Giammar DE. 2015. Synergistic effect of reductive and ligand-promoted dissolution of goethite. Environ. Sci. Technol. 49: 7236-7244.

43. Solanki MK, Singh RK, Srivastava S, Kumar S, Kashyap PL, Srivastava AK, et al. 2014. Isolation and characterization of siderophore producing antagonistic rhizobacteria against Rhizoctonia solani. J. Basic Microbiol. 54: 588-597.

44. Bing Li, Qing Li, Zhihui Xu, Nan Zhang, Qirong Shen, Ruifu Zhang. 2014. Responses of beneficial Bacillus amyloliquefaciens SQR9 to different soilborne fungal pathogens through the alteration of antifungal compounds production. Front Microbiol. 5: 636.

45. Saha, M, Sarkar S, Sarkar B,Sharma BK, Bhattacharjee S, Tribedi P. 2016. Microbial siderophores and their potential applications: a review. Environ. Sci. Pollut. Res. Int. 23: 3984-3999.

46. Ye L, Hildebrand F, Dingemans J, Ballet S, Laus G, Matthijs S, et al. 2014. Draft genome sequence analysis of a Pseudomonas putida W15Oct28 strain with antagonistic activity to Gram-positive and Pseudomonas sp. Pathogens. PLoS One 9: e110038.

47. Yang X, Yousef AE. 2018. Antimicrobial peptides produced by Brevibacillus spp.: structure, classification and bioactivity: a mini review. World J. Microbiol. Biotechnol. 34(4): 57.

48. Che JM, Liu B, Chen Z, Shi H, Liu GD, Ge CB. 2015. Identification of ethylparaben as the antimicrobial substance produced by Brevibacillus brevis FJAT-0809-GLX. Microbiol. Res. 172: 48-56. 